\title{
A Novel Approach to Power Circuit Breaker Design for Replacement of $\mathrm{SF}_{6}$
}

\author{
D. J. Telfer, J. W. Spencer, G. R. Jones, J. E. Humphries
}

This contribution explores the role of PTFE ablation in enhancing current interruption for various background gases in high voltage circuit breakers. An assessment of the current interruption capability has been made in terms of the arcing duration and the contact gap length at which critical arc extinction is achieved. These observations are supported by measurements of the magnitude of extinction and re-ignition voltage peaks. Most previous and other current experimental work on gas filled circuit breaker design follows conventional wisdom in investigating arcing behaviours at elevated gas pressures (usually up to 6 bar). But in this work we concentrate on the effects of using low gas pressures (less than 1 bar) in the presence of a close-fitting shield of ablatant polymer material (PTFE) that surrounds the electrode assembly of an experimental high power circuit breaker. We demonstrate that for several different gases, arc extinction capability compares well under these conditions with $S F_{6}$, suggesting that $S F_{6}$ could be replaced entirely in this novel system by more environmentally friendly gases. Moreover, the critical contact gap lengths at extinction are only slightly greater than when using SF at 6 bar. Weight loss measurements from the ablatant shield suggest that a chemical puffer action is the most likely mechanism for achieving the observed arc extinctions in this system.

Keywords: circuit breaker, power distribution, $S F_{6}$ replacement, arc-induced ablation, anti-pollution.

\section{Introduction}

There have been many attempts to find arc gas environments that might compete with $\mathrm{SF}_{6}$ as an efficient arc-extinguishing medium. Although a range of pure gases and gas mixtures has been investigated [2], a useful combination remains to be identified. This quest has recently been given impetus, fuelled by the realisation that $\mathrm{SF}_{6}$ is one the most potent greenhouse gases known (Christophorou, et al, 1997 [1]). This contribution explores the possibility of effective arc extinction with gases at lower pressures than are usually used, and preferably without $\mathrm{SF}_{6}$.

In this paper we revisit the problem of investigating the arc-extinguishing capabilities of some relatively environmentally friendly gases, and also $\mathrm{SF}_{6}$, but now in a system that has more affinity with puffer type interrupters, an area that has to some extent been hitherto neglected. Particular attention is paid to the possible ablation of PTFE from the flow constraining shield and the manner in which the different gases introduced (,background gases') might affect such ablation effects. Various forms of PTFE sleeve inner linings have been investigated including solid rings and compressed powders. A solid sleeve, honed to a clearance fit without inner lining, was found to perform best, and is the arrangement described in this work.

Judgements regarding the relative effectiveness of the various conditions for promoting arc extinction are made on the basis of a number of factors, which could be conveniently assessed. These have included the measurements of the minimum contact gap length at which current interruption can be achieved and the extent to which arc extinction and re-ignition voltage peaks occur.

The gases investigated are $\mathrm{SF}_{6}, \mathrm{~N}_{2}$, Air, $\mathrm{CO}_{2}$ and Ar. The effect of pressure has also been considered for each of these gases.

\section{Equipment used and experimental approach}

The experimental interrupter unit used in these investigations is shown schematically in Fig. 1 and is based on that of Telfer, et al $[4,5]$. The PTFE shield configuration was fitted to an industrial circuit breaker unit rated at $145 \mathrm{kV}$ and $60 \mathrm{kA}$. The electrode sub-assembly consisted of a hollow fixed contact (copper-tungsten) and a solid cylindrical moving contact (copper-tungsten), which withdrew along a $22 \mathrm{~mm}$ diameter sliding-fit cylindrical channel within the PTFE sleeve. The entire arrangement is housed within a gas tight enclosure. The equipment thus allowed exploration of the role of arc-induced ablation from the close-fitting PTFE cylinder, resulting in enhanced current interruption, for various background gases in high voltage circuit breakers.

The unit was used to interrupt currents produced by a resonant LC circuit [3] at $6.3 \mathrm{kV}$ and tuned to approximately 50 Hz. Peak AC currents up to about $20 \mathrm{kA}$ were investigated. The power source also provided a capability to produce a low level quasi-steady current prior to switching to the full AC fault current mode.

Fig. 1 shows the various timing sequences, ranging from circuit breaker trigger timing (dark square to left of waveform) and capacitor bank dump operation (end of period duration $\mathrm{C}$ ). The approximate position of the interrupter contact at various time instances can be recognised by comparing the time intervals on the current waveform diagram with the corresponding position on the contact displacement diagram. In a typical current waveform produced by the current source, the preliminary quasi-steady current (starting left of A) is followed by two half cycles (B, C) at approximately $50 \mathrm{~Hz}$. As delay A is increased, the waveform in B and C is pushed to the right, until a point is reached where the contacts will have separated too far to support a two half-cycle 


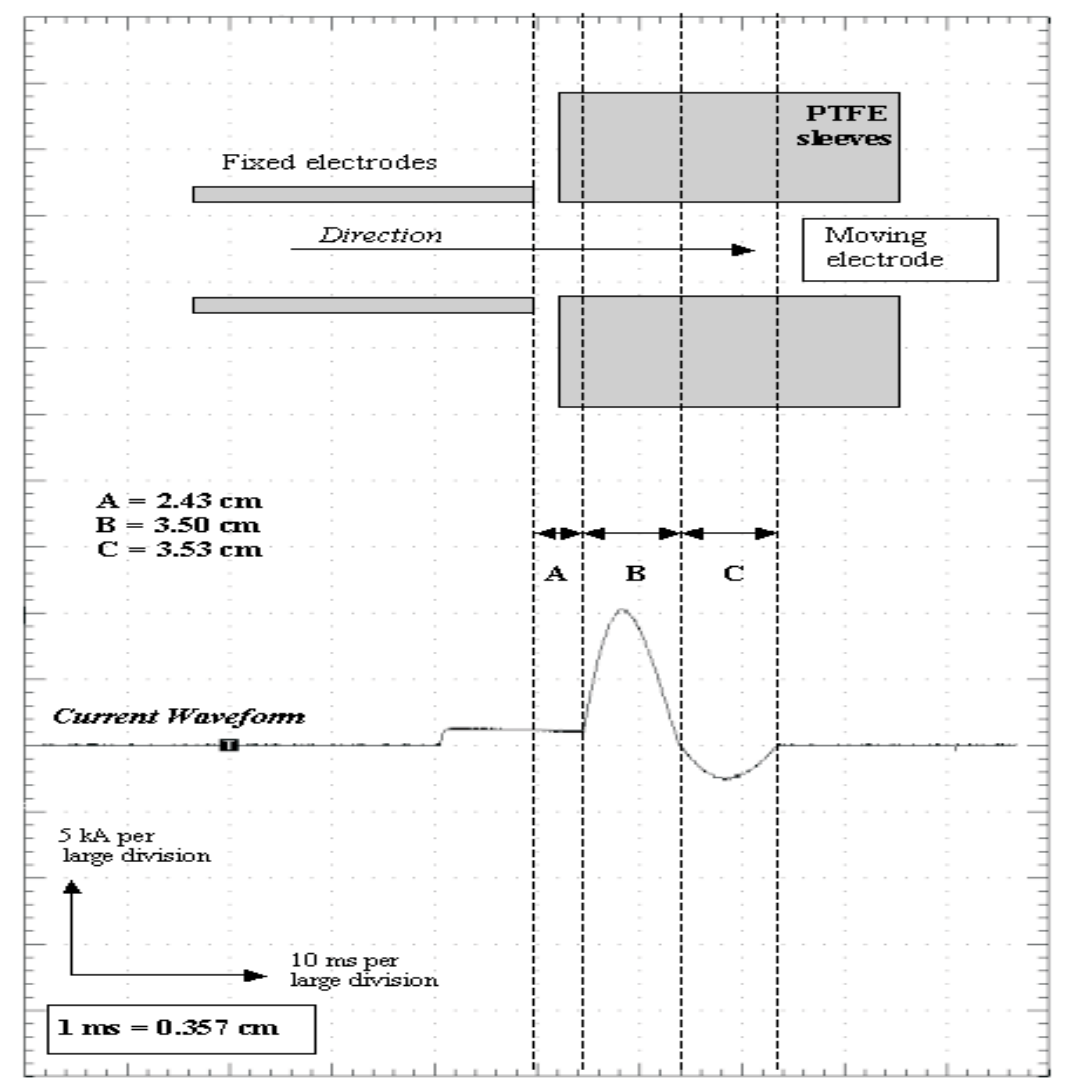

Fig. 1: Relationship between physical distance and current waveform (see text)

regime. At this critical gap and beyond, there is no re-ignition of the arc past current-zero and only one half-cycle of current is sustained. The critical gap (CG), which marks the onset of the single half-cycle regime, is sensitive to arcing conditions including the pressure and nature of the gases present in the arc channel. Thus for a high dielectric strength medium like high pressure ( $>6$ bar) $\mathrm{SF}_{6}$, the value of $\mathrm{CG}$ will be small.

The relationship between timing and distance was ascertained by calibration runs in an open chamber using a high-speed camera. The time-position curve was characteristically S-shaped with a sufficiently linear central region to allow simple conversion between time and distance of electrode travel.

For instance, for the example shown, the contact separation (start of period A) was $2.43 \mathrm{~cm}$ at the point at which the first half cycle was triggered (start of B). The duration of the quasi-steady current period was pre-set as required, as was the peak alternating current.

\section{Results and discussion}

In this work, an assessment of the current interruption capability was made in terms of the arcing duration and the contact gap length at which critical arc extinction was achieved. These critical conditions were indicated by a transition from a two half-cycle to a one half-cycle regime, with CG $(\mathrm{cm})$ bein measured at the onset of the first one half-cycle firing. In Fig. 2 we plot the shortest gap length between moving and fixed contacts needed to successfully interrupt an alter- nating fault current of peak value $20 \mathrm{kA}$ against gas pressure $p$ psi (absolute) in the range 0.8 to 84 psi. Accuracy was estimated to be \pm 0.1 division ( $\pm 1 \mathrm{~mm}$ ) for $\mathrm{CG}$, and for pressure $\pm 2 \%$ at 10 psi degrading to $\pm 20 \%$ at 1 psi.

Fig. 2 shows that there is a sub-range of background gas pressures between 2 to 10 psi for which arc extinction performance improves. In this respect, $\mathrm{SF}_{6}$ maintains its lead, but the other gases are not far behind.

The noticeable features are:

(a) Relatively small dependence upon gas pressure with $\mathrm{SF}_{6}$.

(b) The improved interruption with $\mathrm{N}_{2}$ for $p<7$ psi.

(c) The similar performance of $\mathrm{N}_{2}$ to $\mathrm{SF}_{6}$ for $p<7$ psi.

(d)The similar behaviour of $\mathrm{CO}_{2}$, air to $\mathrm{N}_{2}, \mathrm{SF}_{6}$ for $p<7$ psi.

(e) The generally poorer performance of Ar but none the less showing a similar trend as $\mathrm{N}_{2}$ and $\mathrm{CO}_{2}$.

(f) In this apparatus, there is convergence in performance for $\mathrm{N}_{2}$ and $\mathrm{SF}_{6}$ at higher pressures.

In (f) above, for $\mathrm{SF}_{6}$ and $\mathrm{N}_{2}$ at higher pressures, gas pressure, particularly within the confining space of the cylindrical shield, becomes the dominating influence on arc extinction.

On the other hand, the similar performance of most gases below 7 psi implies the dominance of a common feature believed to be PTFE ablation and subsequent pressurisation due to arc heating of the ablation by-products. Weighing the PTFE billet after some 250 test firings indicates on an average a PTFE weight loss of 0.14 grams per test (for cylinder and moving electrode diameter $2.2 \mathrm{~cm}$ ). The erosion of the PTFE channel wall is significant but not excessive and the per- 


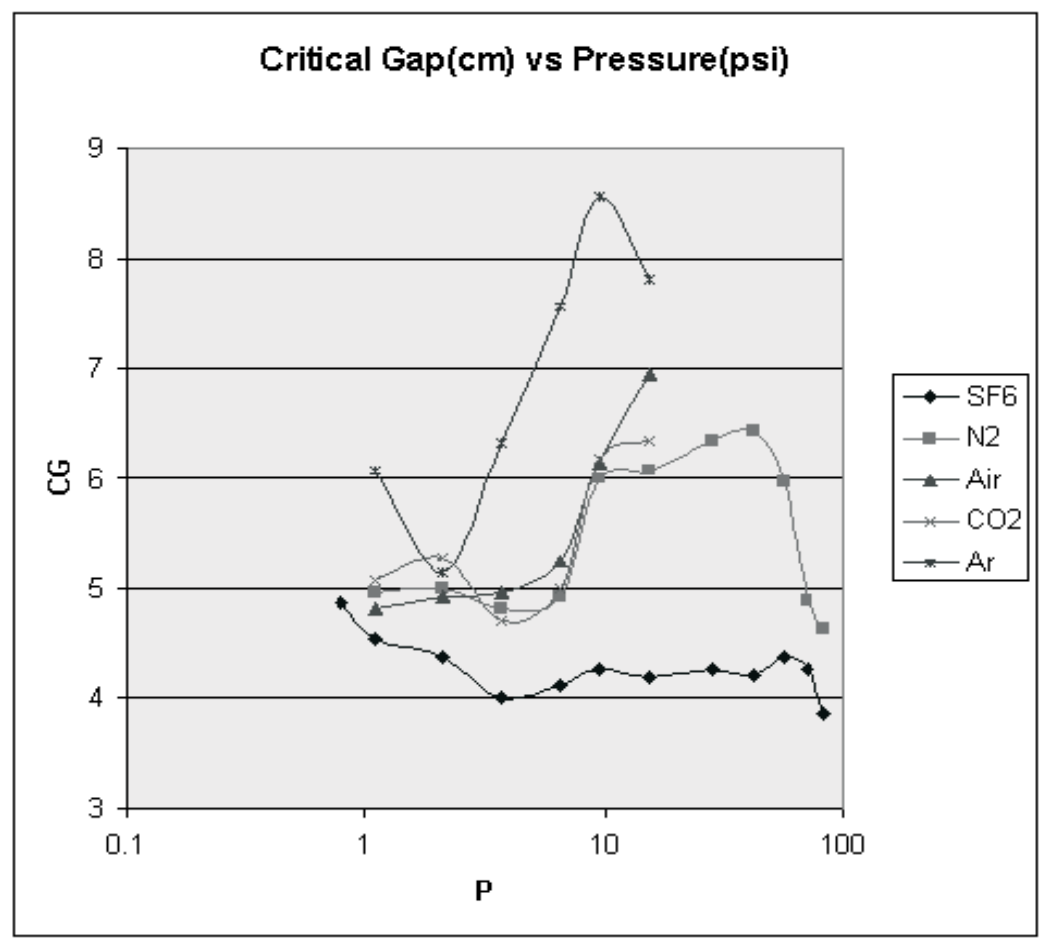

Fig. 2: Critical contact gap length $(\mathrm{cm})$ as a function of background gas pressure (log scale) for a peak alternating current $20 \mathrm{kA}$ with various types of gases $\left(\mathrm{SF}_{6}, \mathrm{~N}_{2}\right.$, Air, $\left.\mathrm{CO}_{2}, \mathrm{Ar}\right)$. Arc extinction performance varies inversely with the value of $\mathrm{CG}$.

formance deteriorates only slightly over 250 tests at fault currents of $20 \mathrm{kA}$ max. Chemical calculations indicated that during these tests, an average of about $30 \%$ of the energy of the arcing process was being used to effect ablation. Thus, irrespective of the ,background gas' being used, transient production of the required chemical species for chemical puffer action is being achieved by arc induced ablation of the polymeric material from the walls of a quasi-cylindrical channel within which the electric arc burns.

Experiments were also conducted to examine the effect of peak alternating current on the critical gap length for current interruption (Fig. 3) at a pressure of 3.7 psi. These show a trend for the interruption performance at lower currents to be approximately at least as effective as at $20 \mathrm{kA}$, as judged by the critical gap length criterion.

Tests were also carried out using a gas mixture, 80:20 $\mathrm{N}_{2}: \mathrm{SF}_{6}$, which behaves in a similar manner to pure $\mathrm{SF}_{6}$ and $\mathrm{N}_{2}$. At the present state of knowledge, there therefore appears to be no significant advantage in utilising $\mathrm{N}_{2}: \mathrm{SF}_{6}$ mixtures in preference to pure $\mathrm{N}_{2}$ unless the recovery of dielectric strength might be improved.

The critical gap length results of Figs. 2 and 3 are supported by measurements of the magnitude of the voltage extinction and re-ignition peaks (as defined in Fig. 4). These

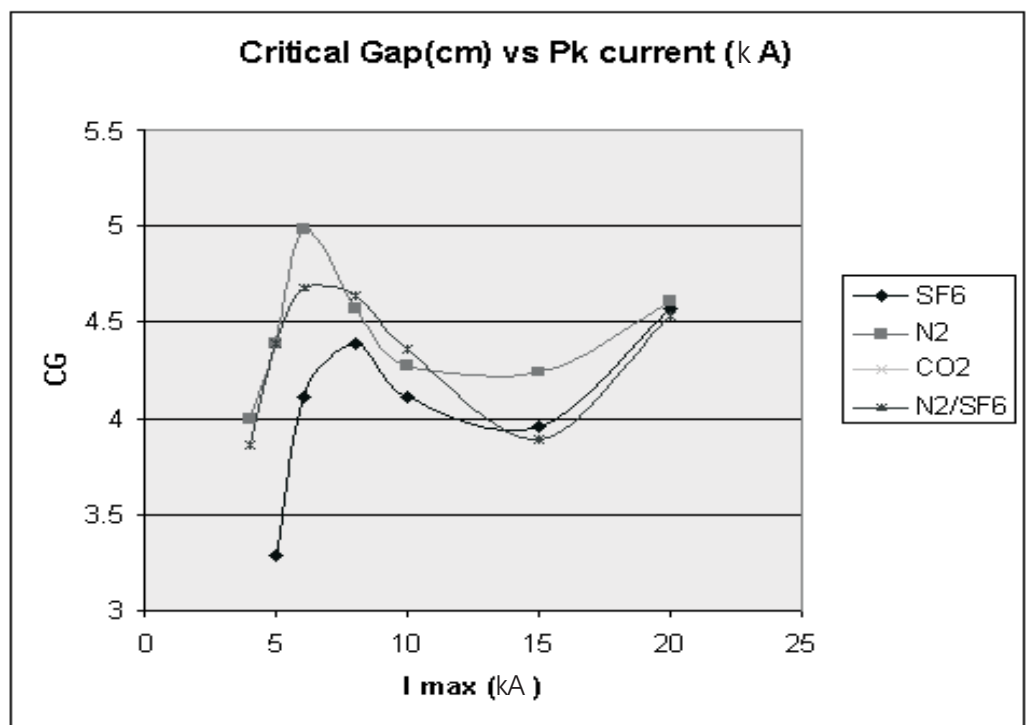

Fig. 3: Critical contact gap length $(\mathrm{cm})$ as a function of maximum fault current with different gases $\left(\mathrm{SF}_{6}, \mathrm{~N}_{2}, \mathrm{CO}_{2}, \mathrm{Ar}\right)$. Arc extinction performance varies inversely with the value of CG. 


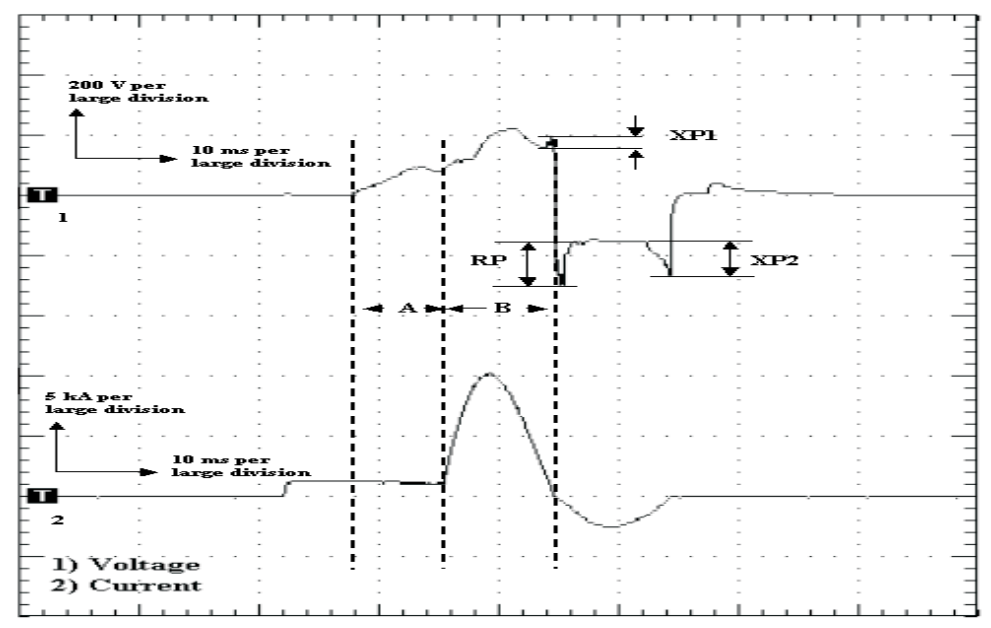

Fig. 4: Example voltage profile (at top) showing extinction and reignition peaks $\left(\mathrm{N}_{2}, 1\right.$ bar)

\section{Extinction and re-ignition peaks, $V$ at $3.7 p s i$}

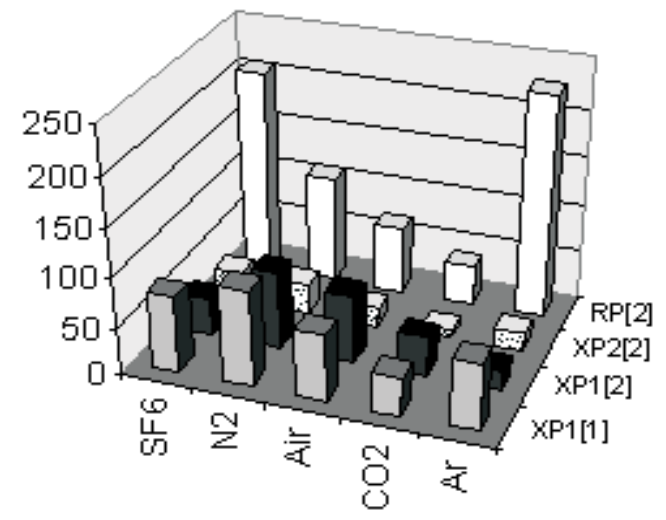

Fig. 5: Extinction and re-ignition peaks with a PTFE cylindrical block, clearance fitting, $145 \mathrm{kV}$ breaker at $20 \mathrm{kA}$ and 3.7 psi $(\mathrm{XP} 1=$ first half-cycle extinction peak, XP2 = second half-cycle extinction peak, RP = second half-cycle re-ignition peak, [1] signifies single half-cycle regime, [2] signifies two half-cycle regime)

were measured close to the critical gap length for current interruption. Fig. 5 compares the relative magnitude of the extinction and re-ignition peaks for a number of gases at a $20 \mathrm{kA}$ and a pressure of 3.7 psi.

The pre-critical extinction and re-ignition peaks results show that under conditions of 3.7 psi at $20 \mathrm{kA}$, the re-ignition peak $(\mathrm{RP})$ in $\mathrm{SF}_{6}(\mathrm{ca} .200 \mathrm{~V})$ is considerably greater $(\sim 2 \times)$ than in $\mathrm{N}_{2}$, but the second half cycle extinction peaks (XP2) in $\mathrm{SF}_{6}$ and $\mathrm{N}_{2}$, although small, are both larger than those for the other gases. And interestingly, the fist half-cycle extinction peaks (XP[1] and XP[2]) for $\mathrm{N}_{2}$ are the larger than for $\mathrm{SF}_{6}$ and the other gases. The reignition peak magnitude when using Ar is relatively high (c.f. also Telfer, et al [4]), in spite of its relatively poor enhancement of performance (Fig. 2). This may possibly be attributable to a difference in prevailing mechanism for this inert gas and is worth further research. Otherwise, the evidence from extinction and re-ignition peak measurements adds credence to the use of $\mathrm{N}_{2}$ as a replacement for $\mathrm{SF}_{6}$ in this type of system.

\section{Summary and conclusions}

An experimental circuit breaker using a close-fitting ablatant shield around the moving electrode has been operated at arc fault currents to $20 \mathrm{kA}$ using various low pressure background gases, including comparison with $\mathrm{SF}_{6}$.

Extinction behaviours using $\mathrm{N}_{2}$, air or $\mathrm{CO}_{2}$ are shown to be similar under these conditions and approach the operating performance when using $\mathrm{SF}_{6}$.

At low pressures below 1 bar (absolute) expansion of the arc flame appears to assist the ablation process. The background gas molecules, along with gases evolved from the ablatant material and dissociation products, contribute to the localised chemical puffer action.

Operation at lower pressures reduces the quantity of $\mathrm{SF}_{6}$, but given the desirability to replace $\mathrm{SF}_{6}$ entirely because of its undesirable 'greenhouse' properties, these results also show that complete replacement of $\mathrm{SF}_{6}$ by $\mathrm{N}_{2}$ at low pressure operation is a viable approach to circuit breaker design. 


\section{Acknowledgment}

The authors appreciate the financial support provided by the Engineering and Physical Sciences Research Council of the UK.

\section{Future work}

Further investigations are warranted to confirm the trends observed and to investigate in more detail the effects of background gas pressure, PTFE configurations, including bore geometries, and weight loss through ablation. Future research will include more quantitative determination of PTFE removal under various controlled conditions. It will also be extended to other appropriate polymeric materials, with suitable background gases and gas pressures, and electromagnetic arc driving forces in order to enhance the production of the suitable chemical species. Efforts are required to establish the optimum combination of these operational conditions that are most likely to produce the required chemical conditions transiently.

\section{References}

[1] Christophorou L. G., Olthoff J. K., Green D. S.: "Gases for Electrical Insulation and Arc Interruption: Possible Present and Future Alternatives to Pure $\mathrm{SF}_{6}$." NIST Tech. Note 1425, 1997, 44 p. (US Dept. of Commerce).
[2] Frind G., Kinsinger R. E., Miller R. D., Nagamatsu T. T., Noeske H. O.: EPRI EL-284, project 246-1, Final report, January 1977.

[3] Furlong S. A.: Characterisation of Electromagnetic Emissions from Circuit Breaker Arcs. PhD. Thesis, The University of Liverpool, 1999.

[4] Telfer D. J., Humphries J., Spencer J. W., Jones G. R.: "Influence of PTFE on Arc Quenching in an Experimental Self-Pressurised Circuit Breaker." Proc $14^{\text {th }}$ Int. Conf. on Arc Discharges and their Applications, Liverpool, 2002.

[5] Telfer D. J., Spencer J. W., Jones G. R., Humphries J.: Patent pending, 2002.

\section{J.Telfer}

e-mail: djtelfer@liverpool.ac.uk

J. W.Spencer

G. R. Jones

J. E. Humphries

Centre for Intelligent Monitoring Systems

Department of Electrical Engineering and Electronics

University of Liverpool

Brownlow Hill,

LIVERPOOL L69 3GJ, UK 